\title{
Hunting Increases Adaptive Auditory Map Plasticity in Adult Barn Owls
}

\author{
Joseph F. Bergan, Peter Ro, Daniel Ro, and Eric I. Knudsen \\ Department of Neurobiology, Stanford University School of Medicine, Stanford, California 94305-5125
}

The optic tectum (OT) of barn owls contains topographic maps of auditory and visual space. Barn owls reared with horizontally displacing prismatic spectacles (prisms) acquire a novel auditory space map in the $0 \mathrm{~T}$ that restores alignment with the prismatically displaced visual map. Although juvenile owls readily acquire alternative maps of auditory space as a result of experience, this plasticity is reduced greatly in adults. We tested whether hunting live prey, a natural and critically important behavior for barn owls, increases auditory map plasticity in adult owls. Two groups of naive adult owls were fit with prisms. The first group was fed dead mice during 10 weeks of prism experience, while the second group was required to hunt live prey for an identical period of time. When the owls hunted live prey, auditory maps shifted substantially farther (five times farther, on average) and the consistency of tuning curve shifts within each map increased. Only a short period of time in each day, during which the two groups experienced different conditions, accounts for this effect. In addition, increased map plasticity correlated with behavioral improvements in the owls' ability to strike and capture prey. These results indicate that the experience of hunting dramatically increases adult adaptive plasticity in this pathway.

Key words: optic tectum; superior colliculus; learning; plasticity; multimodal integration; sound localization

\section{Introduction}

The capacity of the brain to learn from experience is modulated by intrinsic and environmental factors. One powerful intrinsic factor is developmental stage, often expressed as sensitive periods, as exemplified in the development of vision, language, and social behavior (Harlow et al., 1965; Katz, 1999; Newport et al., 2001). Recent studies have shown that environmental factors such as social interactions and environmental richness also modulate plasticity (Newport et al., 2001; Goldstein et al., 2003; Cancedda et al., 2004). Barn owls pass through a similar sensitive period during which experience exerts an exceptionally strong shaping influence on sound localization behavior (Knudsen and Knudsen, 1989b). The effects of experience on sound localization have been traced to specific sites within the owl's midbrain, making it possible to correlate behavioral learning with changes in underlying neural circuits (Feldman and Knudsen, 1998; DeBello et al., 2001).

Owls determine the location of an auditory stimulus from a variety of monaural and binaural cues. The dominant auditory cue for the horizontal location of a stimulus is the timing difference between the sounds arriving at the near and far ears, called interaural timing difference (ITD). The central auditory system measures ITD and associates a particular value of ITD with the

Received June 20, 2005; revised Sept. 8, 2005; accepted Sept. 8, 2005.

J.F.B. was supported by a National Science Foundation Graduate Research Fellowship, and this work was supported by grants to E.I.K. from the National Institutes of Health and the MacArthur Foundation. We thank J. Raymond, D. Moore, K. Maczko, D. Winkowski, and I. Witten for critical comments on this manuscript. We also thank P. Knudsen and B. Linkenhoker for technical assistance.

Correspondence should be addressed to Eric I. Knudsen, Department of Neurobiology, Stanford University School of Medicine, Stanford, CA 94305-5125. E-mail: eknudsen@stanford.edu.

DOI:10.1523/JNEUROSCI.2533-05.2005

Copyright $\odot 2005$ Society for Neuroscience $\quad$ 0270-6474/05/259816-05\$15.00/0 appropriate horizontal location in space. In the optic tectum (OT), these associations are represented topographically as aligned maps of auditory and visual space (Knudsen, 1982). The appropriate alignment of the auditory map with the visual space map in the OT enables the owl to orient its gaze toward salient stimuli regardless of the sensory modality.

Auditory space cues are created by the physical properties of the head and external ears, and the relationships between auditory cue values and spatial locations change as these structures grow (Knudsen et al., 1984). A variety of techniques that change the relationship between auditory cues and visual field locations have been used to investigate how the alignment between auditory and visual space maps develops and is maintained in various species (King et al., 1988; Knudsen and Brainard, 1991; King and Carlile, 1993; Gold and Knudsen, 2000). For example, the relationship between ITD values and locations in visual space can be altered by mounting horizontally displacing prismatic spectacles (prisms) on owls. Immediately after prisms are mounted, an owl will strike an auditory target accurately because the prisms do not alter auditory space cues. However, like many other species, owls quickly recalibrate their movements based on vision (Knudsen and Knudsen, 1989a). Because movements now are calibrated to the prismatically displaced visual field, the owls must also recalibrate their ITD maps to match the visual maps to reestablish an aligned sensorimotor coordinate frame. Young owls do just this, and the recalibration of the auditory map is expressed as a shift of ITD tuning in the OT (Brainard and Knudsen, 1998).

Conditions that lead to near-complete auditory map adjustment in juvenile owls induce little plasticity in adult owls (Brainard and Knudsen, 1998). In one experiment, the owls were fit with prisms at different developmental stages. Prism experience induced large adaptive changes in the auditory maps of juvenile 
owls but induced negligible effects in the maps of adult owls (Brainard and Knudsen, 1998). Similar age dependencies have been described for the plasticity of the auditory space map in the superior colliculus (homolog of the OT) of ferrets and guinea pigs (Withington-Wray et al., 1990; King and Carlile, 1995).

In all previous studies in owls, the plasticity of the space map has been tested in animals that were fed dead food. However, hunting is a behavior that involves precise auditory and visual localization, accurate gaze control, and high levels of attention and arousal. Because each of these factors could influence neural plasticity, we tested whether hunting increases adaptive plasticity in the auditory space map of adult owls.

\section{Materials and Methods}

Animals and surgical preparation. Twelve adult barn owls (Tyto alba) were used in this study. Owls were housed in groups of five and seven in large communal aviaries. All birds were cared for according to the National Institutes of Health Guide for the Care and Use of Laboratory Animals as well as the Stanford University Institutional Animal Care and Use Committee. Two weeks before the start of an experiment, the owls were anesthetized with $1 \%$ halothane mixed with nitrous oxide and oxygen (45\%/55\%). The skull was exposed, and lightweight aluminum prisms were mounted with dental acrylic as described previously (Feldman and Knudsen, 1998). A small stainless-steel fastener was attached to the rear of the skull with dental acrylic to allow for stabilization of the head during electrophysiological recordings.

Hunting epochs were restricted to $1 \mathrm{~h}$ each day. During these sessions, live mice were introduced into the large flight aviary one at a time. The light level in the aviary was set to replicate moonlit conditions, and the floor was covered with shredded paper so that the mice made rustling noises when they moved. Conditions were kept the same during nonhunting epochs except that the owls were fed dead mice. The behavior of each owl was videotaped and monitored remotely.

Electrophysiology. Craniotomies were opened dorsal to the OT, based on stereotaxic coordinates. During recording sessions, the owls were suspended in a prone position with the visual axes aligned with a calibrated screen. A mix of nitrous oxide and oxygen (45\%/55\%) was administered constantly. Multiunit responses were recorded from both the superficial and deep layers of the OT with insulated tungsten microelectrodes ( $6 \mathrm{M} \Omega$ at $1 \mathrm{kHz}$; FHC, Bowdoinham, ME), and high signal-tonoise spikes were isolated with a level discriminator. All recordings were made from the OT on the side of the brain on which the adaptive shift in the ITD map was toward the more contralateral ear leading ITDs (left OT for left-shifting prisms and right OT for right-shifting prisms). When owls exhibit different amounts of shift on the two sides of the brain, this side contains more consistent shifts (Linkenhoker et al., 2005). Spike times were stored by using customized software. Four to six electrode penetrations were made during each experiment, with between two and six multiunit sites recorded on each penetration. Successive recording sites were separated by at least $300 \mu \mathrm{m}$.

Measurements. Visual receptive fields were measured by projecting moving bars of positive or negative contrast on a calibrated screen in front of the owl. The elevation and azimuth (az) of the receptive field center were recorded for each site. ITD tuning was measured by the delivery of $50 \mathrm{~ms}$ broadband $(2-10 \mathrm{kHz} ; 20-30 \mathrm{~dB}$ above threshold) noise bursts dichotically. Auditory stimuli were delivered by using matched Knowles Electronics (Itasca, IL) ED-1914 miniature earphones coupled to dampening assemblies (BF-1743) placed $5 \mathrm{~mm}$ from the ear drum. The amplitude and phase spectra of the earphones were equalized to within $\pm 2 \mathrm{~dB}$ and $\pm 2 \mu \mathrm{s}$, respectively. ITD response curves were generated by sampling at $20 \mu$ s intervals over a $200 \mu$ s range of ITDs approximately centered on the strongest response. Each stimulus set was presented 15-25 times in a randomly interleaved manner. Responses were characterized as the average number of spikes in a $100 \mathrm{~ms}$ window after the onset of the stimulus normalized by the average baseline firing rate. The best tuning was determined by fitting a Gaussian curve to the data with IgorPro (Wavemetrics, Lake Oswego, OR).

Sampling of the OT was restricted to the areas representing -15 to $+15^{\circ}$ elevation and left $20^{\circ}$ to right $20^{\circ}$ relative to the visual axes. This region of visual space is well within the area displaced by the prismatic spectacles. In this region of space, the relationship between the expected best ITD and the horizontal center of the visual receptive field (VRF az) is well described by the following function: expected best ITD $=($ VRF az) $\times\left(2.5 \mu \mathrm{s} /{ }^{\circ}\right)$ in control owls (Brainard and Knudsen, 1998). Deviation from normal ITD tuning (ITD shift) was calculated as the difference between the observed best ITD and the expected best ITD. All behavioral and neurophysiological measurements were made double-blind with respect to each other.

\section{Results}

\section{Effect of hunting on map plasticity}

Figure 1 shows ITD tuning in the OT from a single representative owl (median effect). Before the prism experience, ITD tuning was centered on normal values as predicted by visual receptive field locations (see Materials and Methods) (Fig. 1A,B). The correspondence between auditory and visual tuning was measured a second time after 10 weeks of experience with prisms that displaced the visual field $17^{\circ}$ horizontally (Fig. $1 C, D$ ). Prism experience without hunting induced a small ( $5 \mu$ s) adaptive change in the average ITD tuning relative to the values predicted by the locations of the visual receptive fields (map shift). This map shift was only a small fraction of the $43 \mu$ s shift required to restore alignment between the auditory and the optically displaced visual space maps (indicated by arrows in each panel).

After the first experiment, the prisms were removed for 5 weeks, and the restoration of a normal ITD map was confirmed $(0 \pm 5 \mu$ s; data not shown). Then the prisms that displaced the visual field by an equal amount in the opposite direction were mounted in front of the eyes, and the owls were required to hunt live mice for 10 weeks.

Prism experience while hunting live prey resulted in a map shift of $25 \mu \mathrm{s}$, a much greater shift than occurred in the same bird without hunting ( $p<0.001$; Wilcoxon rank sum test) (Fig. $1 E, F)$. A comparison of the variance in ITD tuning relative to visual tuning measured across recording sites indicated that the prism experience with hunting resulted in a more consistent map shift than without hunting, as is illustrated by the greater variance in tuning seen in Figure $1 D$ compared with Figure $1 F$. This difference was observed in each bird after the prism experience with hunting compared with the prism experience without hunting and was statistically significant across the population $(p<0.05$; paired $t$ test).

Figure $2 A$ shows the ITD map shift for each owl in each condition. The extreme left column summarizes ITD tuning in seven normal owls before the prism experience. After 10 weeks of prism experience without hunting (Fig. $2 \mathrm{~A}$, second column from left), there was a small but significant shift of the map in each bird (median shift, $5 \mu \mathrm{s}$ ), similar to previously reported results for adult owls (Brainard and Knudsen, 1998; Linkenhoker and Knudsen, 2002). Four of the seven owls subsequently were fit with prisms of the opposite direction. After 10 weeks of prism experience with the owls hunting live mice, the median shift was $25 \mu \mathrm{s}$ (Fig. $2 A$, second column from right), a map shift five times greater than was observed without hunting $\left[p<10^{-10}\right.$; two-way ANOVA (owl, condition); $\left.F_{(3,1)}=F_{(7,354)}\right]$. The increase in plasticity with hunting was observed for each owl tested under both of the conditions ( $p<0.001$ for each bird; Wilcoxon rank sum test) (Fig. $2 B$ ).

To determine whether prolonged previous exposure to prisms without hunting was responsible for the increased plasticity seen in the hunting condition, we fit a second group of five owls with prisms and immediately required them to hunt live mice. After 8 
weeks of prism experience with hunting, the owls showed a median map shift of 26 $\mu$ s (Fig. $2 A$, extreme right column). The magnitude of these shifts was similar to that observed in the first group of owls with hunting.

\section{Improvement in strike accuracy} correlates with map plasticity

Over the course of the prism experience, hunting strikes became more accurate. Strike success was measured for four birds as the proportion of strikes for which the mouse was captured (successful strike) from all strike attempts (Fig. 3). Without prisms, the strike success averaged $89 \%$ [95\% confidence interval (CI), 75-97\%]. During the week immediately after prisms were mounted, the strike success rates dropped to an average of $27 \%$ (95\% CI, $20-35 \%)$. After 10 weeks of prism experience with hunting, most birds showed substantial improvement in strike success (Fig. 3). The improvement in strike success during the course of the prism experience correlated with the shift of the ITD map for that bird. Thus, as expected from the logic discussed in the Introduction, adaptive shifts in the auditory map were predictive of improvements in strike accuracy $\left(r^{2}=0.90 ; p<0.05\right)$ (Fig. 3$)$.

\section{Discussion}

Barn owls depend on accurate sound localization to hunt successfully in the dark. We have shown that the act of hunting promotes the adaptive adjustment of the auditory space map in adult owls, maintaining the accuracy of the map required for guiding motor behavior. Hunting increased map plasticity approximately fivefold and improved the consistency of the tuning curve shifts for each owl.

The increase in adult map plasticity caused by hunting is remarkable, considering how subtle the change associated with hunting was in the animal's daily life. There was no obvious difference in the amount of motor activity between the two conditions. During both hunting and nonhunting epochs, the owls were allowed to feed for only $1 \mathrm{~h}$ each day. In both cases, the owls spent only a small portion of this time actively engaged in acquiring food. Furthermore, feeding behaviors for hunting and nonhunting were quite similar, each requiring the owl to orient to the mouse, fly, and strike the target.

It is not the case that simply increasing the duration of the prism experience without hunting would have led to equivalently large map shifts. In previous experiments that used similar conditions without hunting, adult owls experienced prisms for up to 6 months (Brainard and Knudsen, 1998; Knudsen, 1998). These owls exhibited average map shifts of up to $6 \mu$ ITD, and the map shifts they exhibited after 6 months were not greater than the shifts they exhibited after the first 10 weeks of prism experience. Thus there is something special about the hunting experience that drives plasticity particularly effectively.
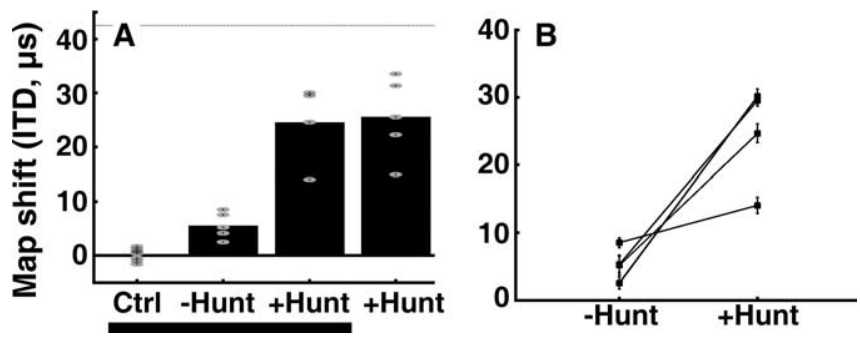

Figure 2. Effect of hunting on auditory plasticity in adult barn owls. $A$, The average map shift in the $0 \mathrm{~T}$ of 12 barn owls. The three left bars represent data from a group of seven owls before prism experience, after prism experience, and after prism experience with hunting (left to right). The extreme right bar represents data from a second group of five owls exposed immediately to hunting with prisms. The maximum expected shift, based on the visual displacement of the prisms, is indicated by the horizontal line at $42.5 \mu \mathrm{s}$. The map shift of each individual bird is indicated for each condition (short horizontal marks). $\boldsymbol{B}$, The map shift \pm SEM that follows prism experience with and without hunting for the four birds for which both conditions were tested. The magnitude of shifts in the hunting and nonhunting conditions was correlated negatively, indicating that larger map shifts induced in the nonhunting condition decreased subsequent shifts to prisms of the opposite direction during the hunting condition (linear regression; $\left.r^{2}=0.94 ; p<0.05\right)$. 


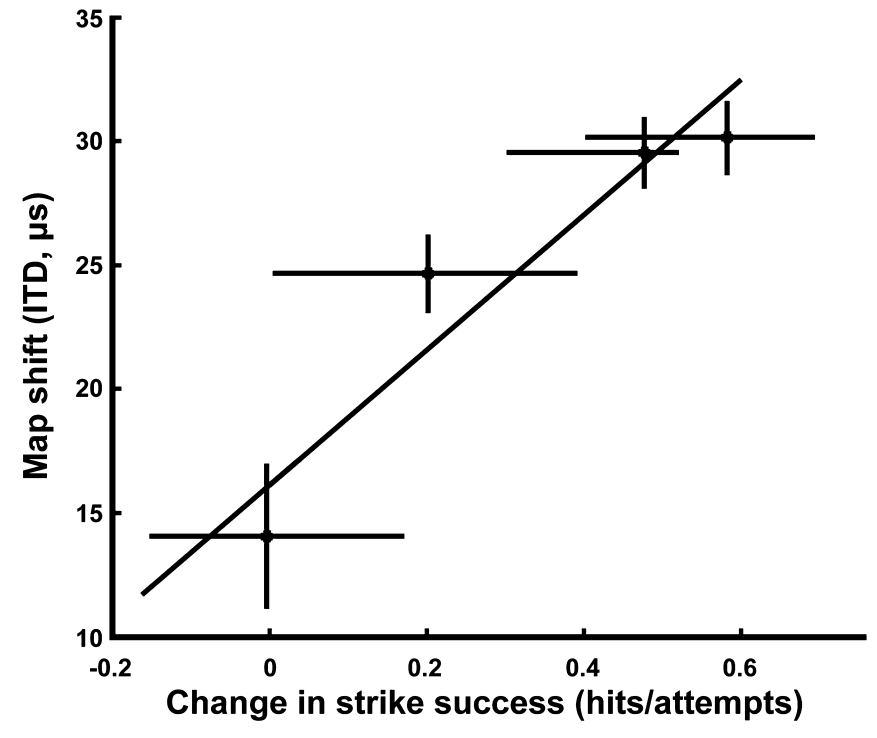

Figure 3. Average map shift of individual owls as a function of the change in strike success. The diagonal line represents the linear correlation of improvement in strike success (final strike success - initial strike success) with the magnitude of auditory map plasticity $\left(r^{2}=0.90 ; p<\right.$ 0.05). Error bars for both strike success and map shift are the $95 \%$ confidence intervals on the measurements.

There were at least four major differences between the hunting and nonhunting conditions. First, hunting was a more difficult task. During hunting epochs, accurate strikes were essential because the mouse would escape otherwise. Second, under hunting conditions the mice made noise. The movements they made, along with spectral and temporal modulations of their noises, helped to capture the owl's attention. Third, live mice provided the owl with both auditory and visual spatial information during moments of heightened attention and arousal, exactly the information required to calibrate the auditory space map. Fourth, accurate cross-modal integration could contribute to the efficient acquisition of food only in the hunting condition.

Factors such as those listed above could increase plasticity in this network via immediate effects associated with the increased sensory information, attention, arousal, and/or reward that accompanied hunting or via long-term changes in the state of the animal (for example, without hunting the owls may have been in a depressed state that inhibited plasticity, and hunting alleviated this state). The immediate effects would act specifically within the hunting epoch, whereas the long-term effects would result in a general increase in the owl's capacity for plasticity. These various influences are not mutually exclusive, and all of them could have contributed to the observed increase in adult plasticity.

\section{Developmental regulation of plasticity}

Although hunting increased map plasticity well beyond that expressed in nonhunting owls, the plasticity exhibited by these adult owls was still substantially less than the plasticity that has been observed in juvenile owls in response to prism experience. Moreover, juvenile owls frequently exhibited full map shifts without hunting and while housed in small individual cages (Knudsen and Knudsen, 1989b). Thus the high degree of plasticity expressed in hunting adult owls is still less than the plasticity expressed by juvenile owls during the sensitive period.

The only experimental manipulation, in addition to hunting, that has enabled adult owls to adapt to large abrupt changes in sensory experience is to have them learn an alternative auditory map during the juvenile sensitive period (Knudsen, 1998). With this background, an adult owl can reacquire a previously learned map. Recent experiments indicate that anatomical circuitry acquired specifically to support the learned map persists into adulthood and could account for the increased capacity for plasticity in these adults. Although these owls are able to switch between normal and previously learned maps, even without hunting, they were not able to learn a new map as adults. These findings indicate that the establishment of a new map of auditory space involves fundamentally different processes than switching between previously established maps. Hunting increases the capacity for adult owls to create new representations of auditory space.

\section{Impact of environmental factors on plasticity}

As demonstrated by this study, certain environmental factors can have a dramatic impact on learning. In previous experiments, juvenile owls have acquired full map shifts without hunting, showing that the sensory information necessary for an owl to acquire a full shift is available in the environment we provided during each condition (Knudsen, 1985). Nevertheless, large adaptive map shifts occurred in adult owls only when they were allowed to hunt. Analogous beneficial effects of environmental factors on learning have been observed for language learning in humans and for song learning in birds (Petrinovich and Baptista, 1987; Goldstein et al., 2003). These findings serve as a reminder that the capacity for learning, and perhaps other sophisticated brain functions, can benefit substantially from the addition of key ethological factors to an animal's environment. Although providing natural conditions often may be difficult or impossible in many laboratory settings, not providing key environmental conditions can alter substantially the quantitative and qualitative outcomes of experiments.

\section{References}

Brainard MS, Knudsen EI (1998) Sensitive periods for visual calibration of the auditory space map in the barn owl optic tectum. J Neurosci 18:3929-3942.

Cancedda L, Putignano E, Sale A, Viegi A, Berardi N, Maffei L (2004) Acceleration of visual system development by environmental enrichment. J Neurosci 24:4840-4848.

DeBello WM, Feldman DE, Knudsen EI (2001) Adaptive axonal remodeling in the midbrain auditory space map. J Neurosci 21:3161-3174.

Feldman DE, Knudsen EI (1998) Pharmacological specialization of learned auditory responses in the inferior colliculus of the barn owl. J Neurosci 18:3073-3087.

Gold JI, Knudsen EI (2000) Abnormal auditory experience induces frequency-specific adjustments in unit tuning for binaural localization cues in the optic tectum of juvenile owls. J Neurosci 20:862-877.

Goldstein MH, King AP, West MJ (2003) Social interaction shapes babbling: testing parallels between birdsong and speech. Proc Natl Acad Sci USA 100:8030-8035.

Harlow H, Dodsworth R, Harlow M (1965) Total social isolation in monkeys. Proc Natl Acad Sci USA 54:90-97.

Katz LC (1999) What's critical for the critical period in visual cortex? Cell 99:673-676.

King AJ, Carlile S (1993) Changes induced in the representation of auditory space in the superior colliculus by rearing ferrets with binocular eyelid suture. Exp Brain Res 94:444-455.

King AJ, Carlile S (1995) Neural coding for auditory space. In: The cognitive neurosciences (Gazzaniga MS, ed), pp 279-293. Cambridge, MA: MIT.

King AJ, Hutchings ME, Moore DR, Blakemore C (1988) Developmental plasticity in the visual and auditory representations in the mammalian superior colliculus. Nature 332:73-76.

Knudsen EI (1982) Auditory and visual maps of space in the optic tectum of the owl. J Neurosci 2:1177-1194.

Knudsen EI (1985) Experience alters spatial tuning of auditory units in the optic tectum during a sensitive period in the barn owl. J Neurosci 5:3094-3109. 
Knudsen EI (1998) Capacity for plasticity in the adult owl auditory system expanded by juvenile experience. Science 279:1531-1533.

Knudsen EI, Brainard MS (1991) Visual instruction of the neural map of auditory space in the developing optic tectum. Science 5:85-87.

Knudsen EI, Knudsen PF (1989a) Visuomotor adaptation to displacing prisms by adult and baby barn owls. J Neurosci 9:3297-3305.

Knudsen EI, Knudsen PF (1989b) Vision calibrates sound localization in developing barn owls. J Neurosci 9:3306-3313.

Knudsen EI, Esterly SD, Knudsen PF (1984) Monaural occlusion alters sound localization during a sensitive period in the barn owl. J Neurosci 4:1001-1011.

Linkenhoker BA, Knudsen EI (2002) Incremental training increases the plasticity of the auditory space map in adult barn owls. Nature 419:293-295.
Linkenhoker BA, von der Ohe CG, Knudsen EI (2005) Anatomical traces of juvenile learning in the auditory system of adult barn owls. Nat Neurosci 8:93-98.

Newport EL, Bavelier D, Neville HJ (2001) Essays in honor of Jacques. In: Language, brain and cognitive development (Doupoux E, ed), pp 481502. Cambridge, MA: MIT.

Petrinovich L, Baptista L (1987) Song development in the white-crowned sparrow: modification of learned song. Anim Behav 35:961-974.

Withington-Wray DJ, Binns KE, Dhanjal SS, Brickley SG, Keating MJ (1990) The maturation of the superior collicular map of auditory space in the guinea pig is disrupted by developmental auditory deprivation. Eur J Neurosci 2:693-703. 